MATHEMATICS OF COMPUTATION

Volume 70, Number 234, Pages 699-703

S 0025-5718(00)01299-0

Article electronically published on October 18,2000

\title{
APPROXIMATION ORDERS FOR NATURAL SPLINES IN ARBITRARY DIMENSIONS
}

TIM GUTZMER AND JENS MARKUS MELENK

\begin{abstract}
Based on variational properties, we generalize the approximation properties of the univariate natural cubic spline to splines in arbitrary dimensions.
\end{abstract}

In one dimension, the solution of the variational problem

$\left\{\right.$ given $a=x_{1}<\cdots<x_{N}=b$ and values $f\left(x_{1}\right), \ldots, f\left(x_{N}\right)$ in $\mathbb{R}$,

$\left\{\right.$ find a function $s$ with $s\left(x_{i}\right)=f\left(x_{i}\right), i=1, \ldots, N$, that minimizes $\left\|s^{\prime \prime}\right\|_{L^{2}([a, b])}$

is given by the natural univariate cubic spline and can be written as (cf. [9])

$$
s(x)=\sum_{i=1}^{N} \lambda_{i}\left|x-x_{i}\right|^{3}+p(x),
$$

where $p(x)$ is a linear polynomial and the coefficients $\lambda_{i}, i=1, \ldots, N$, satisfy the additional constraints

$$
\sum_{i=1}^{N} \lambda_{i}=0, \quad \sum_{i=1}^{N} \lambda_{i} x_{i}=0 .
$$

As shown by Duchon [5] and Meinguet [7], this result can be generalized in a natural way to the $n$-dimensional space $\mathbb{R}^{n}$. This is done in the framework of the Beppo Levi spaces

$$
\mathrm{BL}^{m}(\Omega)=\left\{f \in \mathcal{C}(\Omega), \partial^{\alpha} f \in L^{2}(\Omega) \text { for }|\alpha|=m\right\}
$$

equipped with the inner product

$$
\langle f, g\rangle_{\mathrm{BL}^{m}(\Omega)}=\int_{\Omega} \sum_{|\alpha|=m} \frac{m !}{\alpha !} \partial^{\alpha} f(x) \partial^{\alpha} g(x) d x,
$$

where $\Omega \subset \mathbb{R}^{n}$ is open and $m \in \mathbb{N}$. Here and in the following, $\alpha=\left(\alpha_{1}, \ldots, \alpha_{n}\right) \in \mathbb{N}_{0}^{n}$ denote multi-indices and $|\alpha|=\alpha_{1}+\cdots+\alpha_{n}$. The inner product induces a semi-norm on $\mathrm{BL}^{m}(\Omega)$ via $\|g\|_{\mathrm{BL}^{m}(\Omega)}^{2}=\langle g, g\rangle_{\mathrm{BL}^{m}(\Omega)}$ with polynomial kernel

$$
\mathcal{P}_{m-1}^{n}=\operatorname{span}\left\{x^{\alpha},|\alpha| \leq m-1\right\},
$$

which is a vector space of dimension

$$
M=\left(\begin{array}{c}
n+m-1 \\
n
\end{array}\right) .
$$

Received by the editor July 13, 1999.

2000 Mathematics Subject Classification. Primary 41A15; Secondary 41A63,41A25.

Key words and phrases. Multivariate interpolation. 
Assume that $m>n / 2$ and that $X=\left\{x_{1}, \ldots, x_{N}\right\} \subset \mathbb{R}^{n}, N \geq M$, is a collection of interpolation points that has a $\mathcal{P}_{m-1}^{n}$-unisolvent subset, i.e., there are $\left\{x_{i_{1}}, \ldots, x_{i_{M}}\right\} \subset X$ such that $p\left(x_{i_{j}}\right)=0, j=1, \ldots, M$ for $p \in \mathcal{P}_{m-1}^{n}$ implies $p=0$. The minimal norm interpolant $s_{f}$ on $\mathrm{BL}^{m}\left(\mathbb{R}^{n}\right)$ under the constraints $s_{f}\left(x_{i}\right)=f\left(x_{i}\right), i=1, \ldots, N$, is given by the solution of the variational problem

$$
\left\{\begin{array}{l}
\text { find a function } s_{f} \in \mathrm{BL}^{m}\left(\mathbb{R}^{n}\right) \text { that minimizes }\left\|s_{f}\right\|_{\mathrm{BL}^{m}\left(\mathbb{R}^{n}\right)} \text { and } \\
\text { interpolates } s_{f}\left(x_{i}\right)=f\left(x_{i}\right), i=1, \ldots, N .
\end{array}\right.
$$

It can be written in the form $([\underline{5}, 7])$

$$
s_{f}(x)=\sum_{i=1}^{N} \lambda_{i} E\left(x-x_{i}\right)+p(x),
$$

where $p \in \mathcal{P}_{m-1}^{n}$ and the coefficients $\lambda_{i}, i=1, \ldots, N$, satisfy the constraint

$$
\sum_{i=1}^{N} \lambda_{i} q\left(x_{i}\right)=0 \text { for all } q \in \mathcal{P}_{m-1}^{n}
$$

The function $E$ is the fundamental solution of the iterated Laplacian $\Delta^{m}$ given by

$$
E(x)= \begin{cases}\frac{(-1)^{n / 2+1}}{2^{2 m-1} \pi^{n / 2}(m-1) !(m-n / 2) !}\|x\|_{2}^{2 m-n} \log \|x\|_{2}, & \text { if } 2 m \geq n \text { and } n \text { is even, } \\ \frac{(-1)^{m} \Gamma(n / 2-m)}{2^{2 m} \pi^{n / 2}(m-1) !}\|x\|_{2}^{2 m-n}, & \text { else. }\end{cases}
$$

Remark 1 . If $\Omega \neq \mathbb{R}^{n}$ is a domain, one might think of generalizing the one dimensional result mentioned at the outset by replacing $\mathbb{R}^{n}$ with $\Omega$ in (1). Then, however, one gets representations of the form (2) in which the corresponding function $E$ is the solution of a boundary value problem on $\Omega$ and therefore depends on the geometry of $\Omega$ (cf. Atteia [2]). This is an essential difference to the one dimensional setting.

It is well known [6] that for functions $f \in \mathrm{BL}^{m}(\Omega)$ the minimal norm interpolant $s_{f}$ satisfies

$$
\left\|f-s_{f}\right\|_{L^{\infty}(\Omega)} \leq C h^{m-n / 2}\|f\|_{\mathrm{BL}^{m}(\Omega)}, \quad h=\sup _{x \in \Omega} \inf _{x_{i} \in X}\left\|x-x_{i}\right\|_{2},
$$

where the constant $C$ is independent of $h$ and $f$. The purpose of the present paper is to improve this approximation order by $m$ for functions $f$ from the space

$$
\mathrm{BL}_{0}^{2 m}(\Omega):=\left\{f \in \mathrm{BL}^{2 m}(\Omega), \partial^{\alpha} f=0 \text { on } \partial \Omega \text { for }|\alpha|=m, \ldots, 2 m-1\right\} .
$$

We remark that the boundary conditions are well defined by the trace theorem and the fact that $\mathrm{BL}^{2 m}(\Omega)=H^{2 m}(\Omega)$ algebraically for $\Omega$ open and bounded according to Deny and Lions in [4, Theorem 2.1].

Our result is the following theorem.

Theorem 2. Let $\Omega \subset \mathbb{R}^{n}$ be a bounded domain with Lipschitz boundary. Let $m$, $k \in \mathbb{N}_{0}, p \in[2, \infty]$ with $m>n / 2$ such that the embedding $H^{m}(\Omega) \subset W^{k, p}(\Omega)$ holds. Then there are $C, h_{0}>0$ depending only on $\Omega, m, k$, and $p$ with the following property.

For any collection $X=\left\{x_{1}, \ldots, x_{N}\right\} \subset \Omega$ of interpolation points containing a $\mathcal{P}_{m-1}^{n}$-unisolvent subset and satisfying

$$
h=\sup _{x \in \Omega} \inf _{x_{i} \in X}\left\|x-x_{i}\right\|_{2} \leq h_{0}
$$


and all $f \in \mathrm{BL}_{0}^{2 m}(\Omega)$, the minimal norm interpolant $s_{f}$ given by (1) satisfies

$$
\sum_{|\alpha|=k}\left\|\partial^{\alpha}\left(f-s_{f}\right)\right\|_{L^{p}(\Omega)} \leq C h^{2 m-k-n / 2+n / p}\|f\|_{\mathrm{BL}^{2 m}(\Omega)}
$$

Remark 3. For Lipschitz domains $\Omega$, the embedding $H^{m}(\Omega) \subset W^{k, p}(\Omega)$ holds if (cf. Theorem 5.4 of [1]) the parameters $n, m \in \mathbb{N}, k \in \mathbb{N}_{0}$, and $p \in[2, \infty]$ satisfy

$$
m-k-\frac{n}{2}+\frac{n}{p} \geq 0
$$

except if $n$ is even, in which case the combination $p=\infty$ and $k=m-n / 2$ is excluded.

Remark 4. In one dimension, Theorem 2 recovers the result of 9 for natural cubic splines where the order $7 / 2$ for pointwise estimates is proven.

Remark 5. If one seeks to improve the error bound given by (3) it is not sufficient to increase the regularity of the function $f$. The numerical results in [8] for thin plate spline interpolation in two dimensions $(n=m=2)$ show that even for analytic functions $f$ the order of accuracy is between one and two. It is boundary effects that prevent achievement of higher orders. These boundary effects can be suppressed by imposing boundary conditions on the functions to be approximated as we have done by considering the space $\mathrm{BL}_{0}^{2 m}(\Omega)$.

A different approach to imposing boundary conditions is taken by Schaback in [10]. He uses Fourier techniques and obtains an implicit description of the boundary conditions via a pseudo-differential operator on abstract spaces of functions.

In order to prove Theorem 2, we start with the observation that the minimal norm extensions of elements of $\mathrm{BL}_{0}^{2 m}(\Omega)$ are polynomials outside $\Omega$.

Lemma 6. Let $\Omega \subset \mathbb{R}^{n}$ be a bounded Lipschitz domain, $\mathcal{O}_{1}, \ldots, \mathcal{O}_{\nu}$ be the components of connectedness of $\mathbb{R}^{n} \backslash \bar{\Omega}$, and $f \in B L_{0}^{2 m}(\Omega)$ with $m>n / 2$. Let $f^{\prime}$ be the solution of the problem

$$
\text { minimize }\|u\|_{\mathrm{BL}^{m}\left(\mathbb{R}^{n}\right)} \text { under the constraint }\left.u\right|_{\Omega}=f .
$$

Then there are polynomials $P_{1}, \ldots, P_{\nu} \in \mathcal{P}_{m-1}^{n}$ such that $\left.f^{\prime}\right|_{\mathcal{O}_{i}}=P_{i}, i=1, \ldots, \nu$. Furthermore, $f^{\prime} \in \mathrm{BL}^{2 m}\left(\mathbb{R}^{n}\right) \cap \mathrm{BL}^{m}\left(\mathbb{R}^{n}\right)$ and there holds for all $v \in \mathrm{BL}^{m}\left(\mathbb{R}^{n}\right)$

$$
\left\langle f^{\prime}, v\right\rangle_{\mathrm{BL}^{m}\left(\mathbb{R}^{n}\right)}=\langle f, v\rangle_{\mathrm{BL}^{m}(\Omega)} .
$$

Proof. We start by constructing polynomials $P_{i} \in \mathcal{P}_{m-1}^{n}, i=1, \ldots, \nu$, such that $\left.f\right|_{\partial \mathcal{O}_{i}}=\left.P_{i}\right|_{\partial \mathcal{O}_{i}}$. As $f \in H^{2 m}(\Omega)$ and $\Omega$ is a bounded Lipschitz domain, there is an extension (again denoted $f$ ) to $\mathbb{R}^{n}$ with $f \in H^{2 m}\left(\mathbb{R}^{n}\right)$. By Sobolev's embedding theorem (cf. [1, Theorem 5.4]) in fact $f \in C^{m}\left(\mathbb{R}^{n}\right)$.

Next, the boundaries $\partial \mathcal{O}_{i}$ are components of connectedness of $\partial \Omega$. For each $\partial \mathcal{O}_{i}$ fix one $x_{i} \in \partial \mathcal{O}_{i}$ and define the polynomials $P_{i}$ as Taylor series of order $m$ of $f$ about the point $x_{i}$

$$
P_{i}(x):=\sum_{|\alpha| \leq m-1} \frac{1}{\alpha !} \partial^{\alpha} f\left(x_{i}\right)\left(x-x_{i}\right)^{\alpha} \in \mathcal{P}_{m-1}^{n} .
$$

We claim $f(x)=P_{i}(x)$ for all $x \in \partial \mathcal{O}_{i}$. This follows easily by induction on the differentiation order. By construction there holds

$$
\partial^{\alpha} P_{i}\left(x_{i}\right)=\partial^{\alpha} f\left(x_{i}\right) \quad \forall|\alpha| \leq m .
$$


The induction hypothesis is

$$
\partial^{\alpha} P_{i}(x)=\partial^{\alpha} f(x) \quad \forall x \in \partial \mathcal{O}_{i}, \quad \forall j \leq|\alpha| \leq m .
$$

Equation (6) together with $\partial^{\alpha} f(x)=0$ for $x \in \partial \mathcal{O}_{i}$ and $|\alpha|=m$ shows that the induction hypothesis holds for $j=m$. Supposing that (7) holds for some $j \geq 1$, we conclude that it holds for $j-1$ as follows. Let $\alpha$ with $|\alpha|=j-1$. Then, for $x \in \partial \mathcal{O}_{i}$ and an integration path $\Gamma \subset \partial \mathcal{O}_{i}$, we compute

$$
\partial^{\alpha} f(x)=\partial^{\alpha} f\left(x_{i}\right)+\int_{\Gamma} \nabla \partial^{\alpha} f(y) \cdot d y=\partial^{\alpha} P_{i}\left(x_{i}\right)+\int_{\Gamma} \nabla \partial^{\alpha} P_{i}(y) \cdot d y=\partial^{\alpha} P_{i}(x),
$$

where we made use of (6) and the induction hypothesis; (7) therefore holds for $j=0$.

Define $f^{\prime}$ on $\mathbb{R}^{n}$ by $\left.f^{\prime}\right|_{\Omega}=f,\left.f^{\prime}\right|_{\overline{\mathcal{O}_{i}}}=P_{i}$. Hypothesis (7) implies that $f^{\prime} \in$ $C^{m}\left(\mathbb{R}^{n}\right)$. As $\left\|f^{\prime}\right\|_{\mathrm{BL}^{m}\left(\mathbb{R}^{n}\right)}=\|f\|_{\mathrm{BL}^{m}(\Omega)}$, we conclude $f^{\prime}$ is the solution of (4). Equation (5) is now obvious.

It remains to see that $f^{\prime} \in \mathrm{BL}^{2 m}\left(\mathbb{R}^{n}\right)$. By the form of $f^{\prime}$ on $\mathbb{R}^{n} \backslash \Omega$, it suffices to see that $f^{\prime} \in H_{l o c}^{2 m}\left(\mathbb{R}^{n}\right)$. This follows easily from $\partial^{\alpha} f=0$ on $\partial \Omega$ for $m \leq|\alpha| \leq$ $2 m-1$.

Proof of Theorem Q Let $f^{\prime}$ be the extension of $f$ to $\mathbb{R}^{n}$ given by Lemma 6. By Lemma 6] and an integration by parts (which can be justified by a regularization argument as in [3, Theorems 1.3.4 and 1.6.6]) we obtain using the homogeneous boundary conditions

$$
\left\langle f^{\prime}, f^{\prime}-s_{f}\right\rangle_{\mathrm{BL}^{m}\left(\mathbb{R}^{n}\right)}=\int_{\Omega}\left(\sum_{|\alpha|=m} \frac{m !}{\alpha !} \partial^{2 \alpha} f(x)\right)\left(f(x)-s_{f}(x)\right) d x .
$$

An application of the Cauchy-Schwarz inequality then gives

$$
\left|\left\langle f^{\prime}, f^{\prime}-s_{f}\right\rangle_{\mathrm{BL}^{m}\left(\mathbb{R}^{n}\right)}\right| \leq C\|f\|_{\mathrm{BL}^{2 m}(\Omega)}\left\|f-s_{f}\right\|_{L^{2}(\Omega)},
$$

where $C$ depends only on $n$ and $m$.

Next, from [6, Proposition 3] with $k=0$ and $p=2$, we have

$$
\left\|f-s_{f}\right\|_{L^{2}(\Omega)} \leq C h^{m}\left\|f^{\prime}-s_{f}\right\|_{\mathrm{BL}^{m}\left(\mathbb{R}^{n}\right)}
$$

for some $C>0$ independent of $h$ and $f$. Inserting this in (8) , we obtain with the orthogonality condition $\left\langle f^{\prime}-s_{f}, s_{f}\right\rangle_{\mathrm{BL}^{m}\left(\mathbb{R}^{n}\right)}=0$

$$
\begin{aligned}
\left\|f^{\prime}-s_{f}\right\|_{\mathrm{BL}^{m}\left(\mathbb{R}^{n}\right)}^{2} & =\left\langle f^{\prime}, f^{\prime}-s_{f}\right\rangle_{\mathrm{BL}^{m}\left(\mathbb{R}^{n}\right)} \\
& \leq C\|f\|_{\mathrm{BL}^{2 m}(\Omega)}\left\|f-s_{f}\right\|_{L^{2}(\Omega)} \\
& \leq C h^{m}\|f\|_{\mathrm{BL}^{2 m}(\Omega)}\left\|f^{\prime}-s_{f}\right\|_{\mathrm{BL}^{m}\left(\mathbb{R}^{n}\right)} ;
\end{aligned}
$$

whence we get

$$
\left\|f^{\prime}-s_{f}\right\|_{\mathrm{BL}^{m}\left(\mathbb{R}^{n}\right)} \leq C h^{m}\|f\|_{\mathrm{BL}^{2 m}(\Omega)} .
$$

Finally, combining this with [6. Proposition 3], we get

$$
\begin{aligned}
\sum_{|\alpha|=k}\left\|\partial^{\alpha}\left(f-s_{f}\right)\right\|_{L^{p}(\Omega)} & \leq C h^{m-k-n / 2+n / p}\left\|f^{\prime}-s_{f}\right\|_{\mathrm{BL}^{m}\left(\mathbb{R}^{n}\right)} \\
& \leq C h^{2 m-k-n / 2+n / p}\|f\|_{\mathrm{BL}^{2 m}(\Omega)} .
\end{aligned}
$$




\section{REFERENCES}

[1] Adams, R.A. Sobolev Spaces. 1975 San Diego: Academic Press. MR 56:9247

[2] Atteia, M. Fonctions "spline" et noyaux reproduisants d'Aronszajn-Bergman. R.A.I.R.O. 4 (1970), 31-43. MR 45:9109

[3] Brenner, S.C., Scott, L.R. The Mathematical Theory of Finite Element Methods. 1994 New York: Springer. MR 95f:65001

[4] Deny, J., Lions, J.-L. Les espaces du type de Beppo Levi. Ann. Inst. Fourier 5 (1954), 305-370. MR 17:646a

[5] Duchon, J. Interpolation des fonctions de deux variables suivant le principe de la flexion des plaques minces. R.A.I.R.O. Analyse numérique 10 (1976), 5-12. MR 57:10315

[6] Duchon, J. Sur l'erreur d'interpolation des fonctions de plusieurs variables par les $D^{m}$-splines. R.A.I.R.O. Analyse numérique 12 (1978), 325-334. MR 80j:41052

[7] Meinguet, J. Multivariate interpolation at arbitrary points made simple. Journal of Applied Mathematics and Physics (ZAMP) 30 (1979), 292-304. MR 81e:41014

[8] Powell, M.J.D. The uniform convergence of thin plate spline interpolation in two dimensions. Numerische Mathematik 68 (1994), 107-128. MR 95c:41037

[9] Schaback, R. Radial basis functions viewed from cubic splines. Proceedings of Mannheim Conference 1996 (Nürnberger G., Schmidt J.W., Walz G., eds). 1997 Basel: Birkhäuser. MR 98h:41012

[10] Schaback, R. Improved error bounds for scattered data interpolation by radial basis functions. Math. Comp. 68 (1999) 201-216. MR 99d:41037

Seminar für Angewandte Mathematik, ETH Zürich, CH-8092 Zürich

E-mail address: TGUTZMER@sairgroup.com

Seminar für Angewandte Mathematik, ETH ZürICH, CH-8092 ZÜrich

E-mail address: melenk@sam.math.ethz.ch 